\title{
Opportunistic Power Allocation for Fading Channels with Non-cooperative Users and Random Access
}

\author{
Jun Sun $^{1}$ and Eytan Modiano \\ Laboratory for Information and Decision Systems \\ Massachusetts Institute of Technology \\ \{junsun, modiano\}@mit.edu
}

\begin{abstract}
We present a game-theoretical model of a wireless communication system with multiple competing users sharing a multiaccess fading channel. With a specified capture rule and a limited amount of energy available, a user opportunistically adjusts its transmission power based on its own channel state to maximize the user's own individual throughput. We derive an explicit form of the Nash equilibrium power allocation strategy. Furthermore, this Nash equilibrium power allocation strategy is unique under certain capture rule. We also quantify the loss of efficiency in throughput due to user's selfish behavior. Moreover, as the number of users in the system increases, the total system throughput obtained by using a Nash equilibrium strategy approaches the maximum attainable throughput.
\end{abstract}

\section{INTRODUCTION}

A fundamental characteristic of a wireless, or satellite, network is that the channel over which communication takes place is often time-varying. This variation of channel quality is due to constructive and destructive interference between multipaths and shadowing effects (fading). When multiple users try to communicate with a satellite, one can exploit the channel variation opportunistically by allowing the user with the best channel condition to transmit. This transmission scheme implies the performance of the network is dictated by the best channel state rather than the average one. Hence, the total throughput of such a network tends to increase with number of users.

An important assumption in using this kind scheme is that there is a centralized scheduler who knows each user's channel condition. To get information about user's channel condition, the scheduler will require each user to estimate its channel fading and transmit this information back. As the number of users in the network increases, the

\footnotetext{
${ }^{1}$ This research is supported by NASA Space Communication Project grant number NAG3-2835.
}

delay in conveying user's channel conditions to the scheduler will limit the system's performance. Hence, a distributive multi-access scheme with no centralized scheduler becomes an attractive alternative.

Distributed multi-access schemes such as the aloha random access protocol have long been studied. Recently, a variation on the aloha scheme that takes user's channel state into consideration (channel-aware aloha) has been proposed by [3]. In their formulation, each user only has knowledge of its own channel condition, but no knowledge of the channel fading of the other users sharing the communication link. Capture was not considered in their paper. In [8], the authors studies multiple power level aloha with the objective of maximizing total system throughput when channels are time invariant. In this paper, we allow the satellite to capture packet depending on the received power and assume the channel is a timevarying fading channel. To maximize their own individual throughput subject to the available energy, users opportunistically adjust their transmission power based on their own channel condition. Also, all of the aforementioned work assume that users will implement the same mandated algorithm and behave in a predictable manner. However, in a distributive environment, users may want to change their communication protocols in order to improve their own performance, making it impossible to ensure a particular algorithm will be adopted by all users in the network. Rather than following some mandated algorithm, in this paper users are assumed to act selfishly (i.e., choose their own power allocation strategies) to further their own individual interests.

The communication system considered in this paper consists of multiple users competing to access a satellite, or a base-station. Each user has an average power constraint. Time is slotted. During each time slot, each user chooses a power level for transmission based on the channel state of current slot, which is only known to itself. 
Depending on the capture model and the received power of that user's signal, a transmitted packet may be captured even if multiple users are transmitting at the same slot.

With each user wants to maximize its own expected throughput, we obtain a Nash equilibrium power allocation strategy which determines the optimal transmission power control strategy for each user. Nash equilibrium of a game is a set of strategies (one for each user) from which there is no profitable unilateral deviation. The obtained optimal power control strategy specifies how much power a user needs to use to maximize its own throughput for any possible channel state. Users get different average throughput based on their average power constraint. Hence, this transmission scheme can be viewed as mechanism for providing quality of service (QoS) differentiation; whereby users are given different energy for transmission. The obtained Nash equilibrium power allocation strategy is unique under certain capture rule. When all users have the same energy constraint, we obtained a symmetric Nash equilibrium.

Due to the selfish behavior of individual users, the overall system throughput will be less than that of a system where users employ the same mandated algorithm. This loss in efficiency is also quantified. In the multiple users' case, as the number of user in the system increases, the symmetric Nash equilibrium strategy approaches the optimal algorithm specified by a system designer (i.e., algorithm that results in the largest total system throughput). In this case, there is no loss of efficiency when users employ the symmetric Nash equilibrium.

Game theoretical approaches to Aloha random access problems have been explored by a number of researchers recently (e.g., [2][9]). In [2], the authors characterized the stability region for a slotted Aloha system with multipacket reception and selfish users for the case of perfect information. In [9], the authors considered the problem of a node computing its own optimal channel access rate in a random access network with two-way traffic. In their setting, a node is interested in both receiving as well as transmitting packets. The existence of Nash equilibrium is shown for node without power constraint as well as with battery power constraint. Our work attempts to apply game theory to the access of a wireless fading channel. In particular, we show that the Nash equilibrium strategy derived is well suited to be used as a power control scheme when there is a large number of users in the system. Other papers dealing with the application of game theory to the random access and resource allocation problems in wireless network include [11][15][16].

This paper is organized as follows. In Section II, we describe the communication system. In Section III, we start by presenting the Nash equilibrium strategy pair for the two users game when the channel state is uniformly distributed over $[0,1]$. The uniqueness of the Nash equilibrium strategy is shown under certain capture rule. A symmetric Nash equilibrium is also obtained when users have the same average power constraint. We then explore the Nash equilibrium strategy for general channel state distribution. In section IV, a symmetric Nash equilibrium strategy is derived for the multiple users case. The throughput obtained by using the Nash equilibrium strategy is shown to approach the maximum attainable throughput. Finally, Section V concludes the paper.

\section{Problem Formulation}

We consider a communication environment with multiple users sending data to a single base station or satellite over multiple fading channels. We assume that each user always has data to be sent to the base station. Time is assumed to be discrete, and the channel state for a given user changes according to a known probabilistic model independently over time. The channels between the users and the base station are assumed to be independent of each other. Let $X_{i}$ be a random variable denoting the channel state for the channel between user $i$ and the base station.

When multiple users are transmitting during the same time slot, it is still possible for the receiver to capture one (or more) user's data. The capture model can be described as a mapping from the received power of the transmitting users to the set $\{1, \cdots, n, 0\}$, where 0 indicates no packet is successfully received. In this paper, we are going to investigate two capture models which will be presented in the later sections.

We assume that each individual user is energy constrained. Specifically, each user $i$ has an average amount of energy $e_{i}$ available to itself during each time slot. We assume that the $e_{i}$ values are known to all users, and that users know the distribution of $X_{i}$ 's. However, the exact value of the channel state $X_{i}$ is known to user $i$ only at the beginning of each time slot.

With a given capture model and the energy constraint, the objective for each user is to design a power allocation strategy to maximize its own expected throughput (or probability of success) per time slot subject to the expected or average power constraint. The power allocation strategy will specify how a user will allocate power in every time slot upon observing its channel state. Under power allocation strategy $g_{i}(\cdot)$, user $i$ transmits a packet with power equal to $g_{i}(x)$ when it sees its channel condition in this time slot is $X_{i}=x$. The received power at the base station is denoted as $f_{i}(x)=x \cdot g_{i}(x)$. 
Formally, let $F_{i}$ be the set of continuous and bounded real-valued functions with finite first and second derivative over the support of $X_{i}$. Then, the strategy space for user i (the set of all possible power allocation schemes), say $S_{i}$, is defined as follows:

$$
S_{i}=\left\{g_{i} \in F_{i} \mid E\left[g_{i}\left(X_{i}\right) \leq e_{i}\right\}\right.
$$

\section{Two Users CASE}

We start by investigating users' strategies in a communication system consisting of exactly two users and one base station. The analytical method used in this section will help us in obtaining equilibrium power allocation scheme in the multiple users case. We begin our analysis with the assumption that channel state $X_{i}$ is uniformly distributed over $[0,1]$ for all $i$. The Nash equilibrium power allocation strategy with general channel state distribution is presented in the subsequent section.

Suppose user 1 and user 2 choose their power allocation strategies to be $g_{1}$ and $g_{2}$ respectively. Given a time slot with channel state realization $\left(x_{1}, x_{2}\right)$, user 1 and user 2 will transmit their packets using power levels $g_{1}\left(x_{1}\right)$ and $g_{2}\left(x_{2}\right)$ respectively. The corresponding received power at the base station are $f_{1}\left(x_{1}\right)=x_{1} \cdot g_{1}\left(x_{1}\right)$ and $f_{2}\left(x_{2}\right)=x_{2} \cdot g_{2}\left(x_{2}\right)$. As in [4] and [5], the capture model used in this section is the following: if $\left[x_{1} \cdot g_{1}\left(x_{1}\right)\right] /\left[x_{2} \cdot g_{2}\left(x_{2}\right)\right] \geq K$ where $K \geq 1$, user 1 's packet will be captured. Likewise, user 2's packet will be captured if $\left[x_{2} \cdot g_{2}\left(x_{2}\right)\right] /\left[x_{1} \cdot g_{1}\left(x_{1}\right)\right] \geq K$. Thus, given a power allocation strategy pair $\left(g_{1}, g_{2}\right)$, where $g_{1} \in S_{1}$ and $g_{2} \in S_{2}$, the expected throughput for user 1 is defined as the following:

$$
G_{1}\left(e_{1}, e_{2}\right)=E_{X_{1}, X_{2}}\left[1_{f_{1}\left(X_{1}\right) \geq K \cdot f_{2}\left(X_{2}\right)}\right]
$$

where

$$
1_{f_{1}\left(X_{1}\right) \geq f_{2}\left(X_{2}\right)}= \begin{cases}1 & \text { if } f_{1}\left(X_{1}\right) \geq K \cdot f_{2}\left(X_{2}\right) \\ 0 & \text { otherwise }\end{cases}
$$

Similarly, the throughput function for user 2:

$$
G_{2}\left(e_{1}, e_{2}\right)=E_{X_{1}, X_{2}}\left[1_{f_{2}\left(X_{2}\right)>K \cdot f_{1}\left(X_{1}\right)}\right]
$$

\section{A. Nash equilibrium strategy}

In this part, we present a Nash equilibrium power allocation strategy pair $\left(g_{1}^{*}, g_{2}^{*}\right)$. A strategy pair $\left(g_{1}^{*}, g_{2}^{*}\right)$ is said to be in Nash equilibrium if $g_{1}^{*}$ is the best response for user 1 to user 2's strategy $g_{2}^{*}$, and $g_{2}^{*}$ is the best response for user 2 to user 1's strategy $g_{1}^{*}$. We consider here the case where both users choose their strategies from the strategy space $S_{1}$ and $S_{2}$ and the value of $e_{1}$ and $e_{2}$ are known to both users.

To get the Nash equilibrium strategy pair, we first argue that at equilibrium the received power function $f_{i}^{*}\left(x_{i}\right)$ must be strictly increasing in $x_{i}$.

Lemma 1: Given a Nash equilibrium power allocation strategy pair $\left(g_{1}^{*}, g_{2}^{*}\right)$ and its corresponding received power function $\left(f_{1}^{*}, f_{2}^{*}\right)$, the received power function $f_{1}^{*}\left(x_{1}\right)$ must be strictly increasing in $x_{1}$. Similarly, $f_{2}^{*}\left(x_{2}\right)$ must be strictly increasing in $x_{2}$.

Proof: For an arbitrary received power function $f$ which is not strictly increasing, we can always find another received power function that will result in a larger throughput gain. To see this, consider time slots with channel state in the small intervals $(a-\delta, a+\delta)$ and $(b-\delta, b+\delta)$ where $a<b$. When $\delta$ is small, the received power function is close to $f(a)$ for time slots in the interval $(a-\delta, a+\delta)$. Likewise, the received power function is close to $f(b)$ for time slots in the interval $(b-\delta, b+\delta)$.

For received power function $f$ such that $f(a)=a$. $g(a)>f(b)=b \cdot g(b)$ for some $a<b$. The total amount of transmission power used in time slots with channel state in the two intervals is given by:

$$
[g(a)+g(b)] 2 \delta=\left[\frac{f(a)}{a}+\frac{f(b)}{b}\right] 2 \delta .
$$

Now, if user 1 employs a new power allocation strategy $\bar{g}$ such that $\bar{g}(b)=\frac{f(a)}{b}$ and $\bar{g}(a)=\frac{f(b)}{a}$, user 1 will achieve the same expected throughput as before. However, the amount of power used $[\bar{g}(b)+\bar{g}(a)] 2 \delta$ is less than $[g(a)+g(b)] 2 \delta$, and the extra power can be used to get higher throughput. Hence, both equilibrium received power function $f_{1}^{*}\left(x_{1}\right)$ and $f_{2}^{*}\left(x_{2}\right)$ must be strictly increasing in $x_{1}$ and $x_{2}$ respectively.

With one user's power allocation strategy, say $g_{2}$, fixed, we now seek the optimal power allocation scheme for user 1. From Lemma 1, we see that the inverse of $f_{1}$ and $f_{2}$ are well defined. With user 2's strategy $g_{2}$ fixed, let $u_{g_{2}}^{(1)}$ : $\left(x_{1}, b\right) \rightarrow \mathcal{R}$ denote user 1's expected throughput of a slot conditioning on the following events:

- User 1's channel state is $X_{1}=x_{1}$.

- User 1's allocated power is $b$.

For convenience, we will drop the term $g_{2}$ in the expression $u_{g_{2}}^{(1)}\left(x_{1}, b\right)$, and simply write it as $u_{1}\left(x_{1}, b\right)$. Specifically, we can the write the equation:

$$
u_{1}\left(x_{1}, b\right)=P\left(f_{2}\left(X_{2}\right) \cdot K \leq x_{1} \cdot b\right)
$$

where $P\left(f_{2}\left(X_{2}\right) \cdot K \leq x_{1} \cdot b\right)$ is the probability that user 1 's packet gets captured in a time slot. Consequently, us- 
ing a strategy $g_{1}$, user 1's throughput is given by:

$$
\begin{aligned}
G_{1}\left(e_{1}, e_{2}\right) & =\int_{0}^{1} u_{1}\left(x_{1}, g_{1}\left(x_{1}\right)\right) \cdot p_{X_{1}}\left(x_{1}\right) d x_{1} \\
& =\int_{0}^{1} u_{1}\left(x_{1}, g_{1}\left(x_{1}\right)\right) d x_{1} .
\end{aligned}
$$

where the last equality results from the uniform distribution assumption.

With user 1's strategy $g_{1}$ fixed, similar terms for user 2 can be defined.

$$
u_{2}\left(x_{2}, b\right)=u_{g_{1}}^{(2)}\left(x_{2}, b\right)=P\left(f_{1}\left(X_{1}\right) \cdot K \leq x_{2} \cdot b\right)
$$

Then, user 2's throughput is given by:

$$
\begin{aligned}
G_{2}\left(e_{1}, e_{2}\right) & =\int_{0}^{1} u_{2}\left(x_{2}, g_{2}\left(x_{2}\right)\right) \cdot p_{X_{2}}\left(x_{2}\right) d x_{2} \\
& =\int_{0}^{1} u_{2}\left(x_{2}, g_{2}\left(x_{2}\right)\right) d x_{2} .
\end{aligned}
$$

Due to the uniformly distributed channel state, $P\left(f_{2}\left(X_{2}\right) \cdot K \leq x_{1} \cdot b\right)$ is given by

$$
\begin{aligned}
P\left(f_{2}\left(X_{2}\right) \cdot K \leq x_{1} \cdot b\right) & =P\left(X_{2} \leq f_{2}^{-1}\left(\frac{1}{K} x_{1} \cdot b\right)\right) \\
& =f_{2}^{-1}\left(\frac{1}{K} x_{1} \cdot b\right)
\end{aligned}
$$

where $f_{2}^{-1}$ is well defined. Thus, we can rewrite Eq. (4) as

$$
u_{1}\left(x_{1}, b\right)=f_{2}^{-1}\left(\frac{1}{K} x_{1} \cdot b\right) .
$$

Hence we have,

$$
\begin{aligned}
& G_{1}\left(e_{1}, e_{2}\right)=\int_{0}^{1} f_{2}^{-1}\left(\frac{1}{K} x_{1} \cdot g_{1}\left(x_{1}\right)\right) d x_{1} \\
& G_{2}\left(e_{1}, e_{2}\right)=\int_{0}^{1} f_{1}^{-1}\left(\frac{1}{K} x_{2} \cdot g_{2}\left(x_{2}\right)\right) d x_{2}
\end{aligned}
$$

We begin our analysis of the Nash equilibrium strategy pair by first considering the power allocation on the boundary points 0 and 1 . For a pair of power allocation functions $\left(g_{1}^{*}, g_{2}^{*}\right)$ to be a Nash equilibrium, it is straightforward to see that $g_{1}^{*}(0)=g_{2}^{*}(0)=0$ since it does not make sense to allocate power for a slot with zero channel state. Likewise, we must have $g_{1}^{*}(1) \leq K \cdot g_{2}^{*}(1)$ and $g_{2}^{*}(1) \leq K \cdot g_{1}^{*}(1)$ since allocating power $g_{1}(1)=K g_{2}(1)$ or $g_{1}(1)=K g_{2}(1)+\epsilon$, where $\epsilon>0$, will result in the same throughput for user 1 . We call these properties the boundary conditions of a Nash equilibrium strategy pair.

With the boundary conditions satisfied, the following lemma gives a necessary and sufficient condition for a pair of power allocation strategies to be a Nash equilibrium strategy pair. For convenience, we denote the marginal gain for user 1 when $X_{1}=x_{1}$ and the allocated power $b=b^{*}$ as

$$
\left.\frac{\partial u_{1}\left(x_{1}, b\right)}{\partial b}\right|_{b=b^{*}} \triangleq D u_{1}\left(x_{1}, b^{*}\right) .
$$

Lemma 2: Given a power allocation strategy pair $\left(g_{1}^{*}, g_{2}^{*}\right)$ that satisfies the boundary conditions, $\left(g_{1}^{*}, g_{2}^{*}\right)$ is a Nash equilibrium strategy pair if and only if $D u_{1}\left(x_{1}, g_{1}^{*}\left(x_{1}\right)\right)=c_{1}$ and $D u_{2}\left(x_{2}, g_{2}^{*}\left(x_{2}\right)\right)=c_{2}$, for some constants $c_{1}$ and $c_{2}$, for all $x_{1} \in[0,1]$ and all $x_{2} \in[0,1]$.

Note that the above lemma does not depend on the assumption of the uniformly distributed channel state. Thus, it is quite general and will be used in the subsequent section where channel states are not uniformly distributed. To understand the lemma intuitively, suppose there exists $x \neq \tilde{x}$ such that $D u_{1}\left(x, g_{1}^{*}(x)\right)>D u_{1}\left(\tilde{x}, g_{1}^{*}(\tilde{x})\right)$. Reducing the power allocated at $\tilde{x}$ to $g_{1}^{*}(\tilde{x})-\delta$ and increasing the power at $x$ to $g_{1}^{*}(x)+\delta$ will result in an increase in the throughput by $\left(D u_{1}\left(x, g_{1}^{*}(x)\right)-D u_{1}\left(\tilde{x}, g_{1}^{*}(\tilde{x})\right)\right) \cdot \delta$. Thus, user 1 has an incentive to change its allocation function, and $\left(g_{1}^{*}, g_{2}^{*}\right)$ cannot be a Nash equilibrium strategy pair in this case.

Proof: The complete proof is given in the Appendix.

With Lemma 2, we are able to find the Nash equilibrium strategy pair. The exact form of the equilibrium power allocation strategies are presented in the following Theorem.

Theorem 1: Given the average power constraint $e_{1}$ and $e_{2}$, the Nash equilibrium power allocation strategy pair has the following form:

$$
\begin{aligned}
& g_{1}^{*}(x)=c_{1} \cdot x^{\gamma} \\
& g_{2}^{*}(x)=c_{2} \cdot x^{\frac{1}{\gamma}}
\end{aligned}
$$

where the constants $c_{1}, c_{2}$ and $\gamma$ are chosen such that

$$
\begin{aligned}
& \int_{0}^{1} c_{1} \cdot x^{\gamma} d x=e_{1} \\
& \int_{0}^{1} c_{2} \cdot x^{\frac{1}{\gamma}} d x=e_{2}
\end{aligned}
$$

Equations (11) and (12) impose the average power constraints.

Proof: We show here that $g_{1}^{*}(x)=c_{1} \cdot x^{\gamma}$ and $g_{2}^{*}(x)=c_{2} \cdot x^{\frac{1}{\gamma}}$ is indeed a Nash equilibrium strategy pair by using the sufficiency condition of Lemma 2 . Since both functions are strictly increasing, we can write $u_{1}(x, b)=f_{2}^{*-1}\left(\frac{1}{K} x \cdot b\right)$ and $u_{2}(x, b)=f_{1}^{*-1}\left(\frac{1}{K} x \cdot b\right)$ 
where $f_{i}^{*}(x)=x \cdot g_{i}^{*}(x)$. Also, since both $f_{1}^{*}$ and $f_{2}^{*}$ are differentiable, we have $u_{1}(x, b)$ and $u_{2}(x, b)$ both differentiable with respect to $b$. Therefore, using

$$
\begin{gathered}
f_{2}^{* \prime}(x)=c_{2}\left(1+\frac{1}{\gamma}\right) x^{\frac{1}{\gamma}}, f_{1}^{* \prime}(x)=c_{1}(1+\gamma) x^{\gamma} \\
f_{2}^{*-1}(x)=\left(\frac{1}{c_{2}} x\right)^{\frac{\gamma}{1+\gamma}}, f_{1}^{*-1}(x)=\left(\frac{1}{c_{1}} x\right)^{\frac{1}{1+\gamma}}
\end{gathered}
$$

we have

$$
\begin{aligned}
& \left.\frac{\partial u_{1}(x, b)}{\partial b}\right|_{b=g_{1}^{*}(x)}=\frac{\frac{1}{K} x}{f_{2}^{* \prime}\left(f_{2}^{*-1}\left(\frac{1}{K} x g_{1}^{*}(x)\right)\right)} \\
& =\frac{\frac{1}{K} x}{f_{2}^{* \prime}\left(\left[\frac{c_{1}}{K c_{2}}\right]^{\frac{\gamma}{1+\gamma}} x^{\gamma}\right)}=\frac{\frac{1}{K}}{c_{2}\left(1+\frac{1}{\gamma}\right)\left(\frac{c_{1}}{K c_{2}}\right)^{\frac{1}{1+\gamma}}} .
\end{aligned}
$$

Similarly,

$$
\begin{aligned}
& \left.\frac{\partial u_{2}(x, b)}{\partial b}\right|_{b=g_{2}^{*}(x)}=\frac{\frac{1}{K} x}{f_{1}^{* \prime}\left(f_{1}^{*-1}\left(\frac{1}{K} x g_{2}^{*}(x)\right)\right)} \\
& =\frac{\frac{1}{K} x}{f_{1}^{* \prime}\left(\left[\frac{c_{2}}{K c_{1}}\right]^{\frac{1}{1+\gamma}} x^{1 / \gamma}\right)}=\frac{\frac{1}{K}}{c_{1}(1+\gamma)\left(\frac{c_{2}}{K c_{1}}\right)^{\frac{\gamma}{1+\gamma}}} .
\end{aligned}
$$

From Lemma 2, we see that $\left(f_{1}^{*}, f_{2}^{*}\right)$ is indeed a Nash equilibrium strategy pair because both $D u_{1}\left(x, g_{1}^{*}(x)\right)$ and $D u_{2}\left(x, g_{2}^{*}(x)\right)$ are constants.

From the above theorem, we see that equations (9) and (10) specify the Nash equilibrium power allocation strategy pair. Since there are two equations with three unknowns, the resulting Nash equilibrium may not be unique in general. However, if a packet with stronger received power can always be captured (i.e., $K=1$ ), the Nash equilibrium power allocation strategy is unique.

Corollary 1: For $K=1$, the unique Nash equilibrium power allocation pair has the following form:

$$
\begin{aligned}
g_{1}^{*}(x) & =c \cdot x^{\gamma} \\
g_{2}^{*}(x) & =c \cdot x^{\frac{1}{\gamma}}
\end{aligned}
$$

where the constants $c$ and $\gamma$ are chosen such that the average power constraints are satisfied.

To show the corollary, we first present the following Lemma.

Lemma 3: If $\left(g_{1}^{*}, g_{2}^{*}\right)$ is a Nash equilibrium strategy pair, $g_{1}^{*}(1)=g_{2}^{*}(1)$.

Proof: $\quad$ Suppose $g_{1}^{*}(1) \neq g_{2}^{*}(1)$. Without loss of generality, let assume that $g_{1}^{*}(1)>g_{2}^{*}(1)$. Since both $g_{1}^{*}$ and $g_{2}^{*}$ are continuous, there exists $\delta>0$ such that $g_{1}^{*}(x)>g_{2}^{*}(1)+\frac{g_{1}^{*}(1)-g_{2}^{*}(1)}{2} \quad \forall x \in[1-\delta, 1]$. User 1 can devise a new allocation strategy, say $\overline{g_{1}}$, by moving a small amount of power, say $\delta \cdot \frac{g_{1}^{*}(1)-g_{2}^{*}(1)}{2}$, away from the interval $[1-\delta, 1]$ to some other interval, thus resulting in an increase in user 1's throughput. Therefore, when $g_{1}^{*}(1)>g_{2}^{*}(1)$, the power allocation strategy pair $\left(g_{1}^{*}, g_{2}^{*}\right)$ cannot be in equilibrium since the strategy pair $\left(\overline{g_{1}}, g_{2}^{*}\right)$ gives a higher throughput for user 1 . Similar result holds for the case $g_{2}^{*}(1)>g_{1}^{*}(1)$. Thus, we must have $g_{1}^{*}(1)=$ $g_{2}^{*}(1)$ if $\left(g_{1}^{*}, g_{2}^{*}\right)$ is an equilibrium strategy pair.

The condition that $g_{1}^{*}(1)=g_{2}^{*}(1)$ will be useful in proving the uniqueness of the Nash equilibrium. The complete proof of the corollary is shown in the appendix.

Fig. 1 shows an example of the Nash equilibrium power allocation strategy pair when $e_{1}=1$ and $e_{2}=2$. Since user 1 has less average power than user 2 , user 1 concentrates its power on time slots with very good channel state. Fig. 2 shows the capture result when both users em-
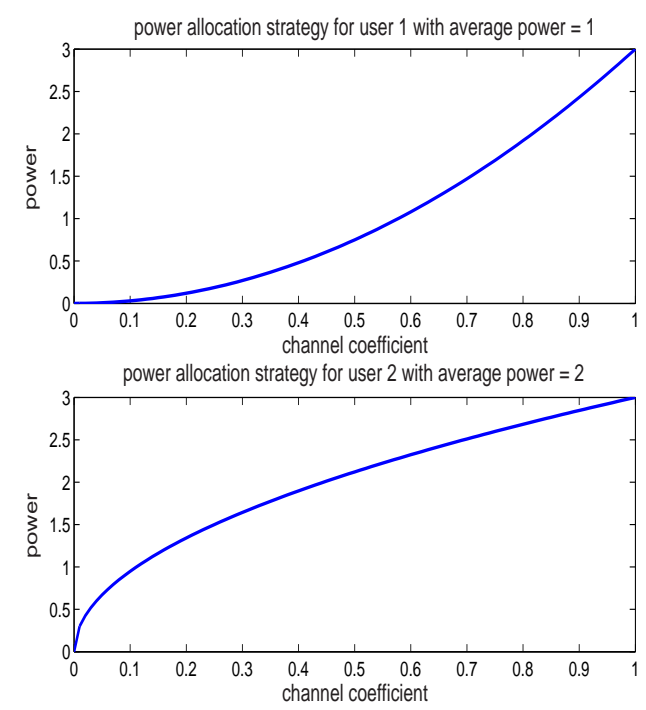

Fig. 1. An example of Nash equilibrium strategy pair for $e_{1}=1$ and $e_{2}=2$.

ploy the Nash equilibrium strategy shown in Fig. 1. For a time slot with channel state realization that fall into the region above the curve, user 2's packet will be successfully captured since user 2's received power is higher than that of user 1 in this region. Here, user 2 has more successful transmissions than user 1 since it has more power.

\section{B. General Channel State Distribution}

In this section, we specify the conditions that a general channel state distribution has to satisfy in order for a Nash equilibrium strategy pair to exist.

From Lemma 1, one can see that $f_{1}$ and $f_{2}$ have to be increasing functions regardless of the distribution of the $X_{i}$ 's. Let $p_{X_{i}}(\cdot)$ denote the probability density function of $X_{i}$ with the support over an interval starting at zero. Assuming $K=1$, the probability that user 1's packet will 


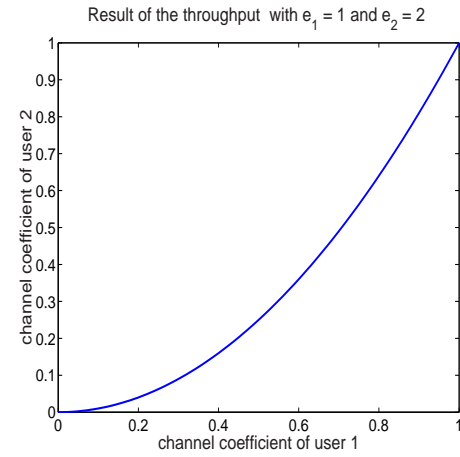

Fig. 2. Results obtained when using the Nash equilibrium strategy pair for $e_{1}=1$ and $e_{2}=2$.

be captured in a time slot with $X_{1}=x_{1}$ and $g_{1}\left(x_{1}\right)=b$ can be written as the following:

$$
\begin{aligned}
u_{1}\left(x_{1}, b\right) & =P\left(f_{2}\left(X_{2}\right) \leq x_{1} \cdot b\right) \\
& =P\left(X_{2} \leq f_{2}^{-1}\left(x_{1} \cdot b\right)\right) \\
& =\int_{0}^{f_{2}^{-1}\left(x_{1} \cdot b\right)} p_{X_{2}}\left(x_{2}\right) d x_{2}
\end{aligned}
$$

From the optimality condition stated in Lemma 2, we must have $D u_{1}\left(x_{1}, b\right)=c_{1}$ where $c_{1}$ is some constant. This condition can be expanded as follows:

$$
\frac{\partial u_{1}\left(x_{1}, b\right)}{\partial b}=p_{X_{2}}\left(f_{2}^{-1}\left(x_{1} \cdot b\right)\right) \frac{x_{1}}{f_{2}^{\prime}\left(f_{2}^{-1}\left(x_{1} b\right)\right)}=c_{1}
$$

Now, let's focus on finding a symmetric Nash equilibrium power allocation strategy. Substituting $b=g_{1}\left(x_{1}\right)$, the term $f_{2}^{-1}\left(x_{1} \cdot b\right)$ is equal to $f_{2}^{-1}\left(f_{1}\left(x_{1}\right)\right)=x_{1}$ since $f_{1}=$ $f_{2}$. Thus, Eq.(16) can be reduced to the following:

$$
\begin{aligned}
& p_{X_{2}}\left(x_{1}\right) \frac{x_{1}}{f_{2}^{\prime}\left(x_{1}\right)}=c_{1} \\
\Rightarrow & f_{2}^{\prime}\left(x_{1}\right)=\frac{1}{c_{1}} x_{1} \cdot p_{X_{2}}\left(x_{1}\right)
\end{aligned}
$$

The above equation provides a condition on the distribution of the $X_{i}$ such that there exists a Nash equilibrium power allocation scheme. The condition can be restated as the following:

$$
x_{1} \cdot g_{1}\left(x_{1}\right)=\int \frac{1}{c_{1}} x_{1} \cdot p_{X_{2}}\left(x_{1}\right) d x_{1}
$$

From the above condition, for example, we see that if $p_{X_{2}}(\cdot)$ is a strictly increasing polynomial, there exist a Nash equilibrium power allocation strategy.

\section{Multiple Users Equilibrium Strategies}

In this section, we explore the Nash equilibrium power allocation strategies when $n$ users are competing to access the single base station. User $i$ 's power allocation function is denoted as $g_{i}(\cdot)$. Given a time slot with channel state realization $\vec{x}=\left(x_{1}, \cdots, x_{n}\right)$, the transmitting power for each user is $g_{i}\left(x_{i}\right)$. The corresponding received power at the base station is again denoted as $f_{i}\left(x_{i}\right)=x_{i} \cdot g_{i}\left(x_{i}\right)$. The new capture rule used in this section is given as the following: a packet from user 1 will be successfully received if the following holds:

$$
f_{1}\left(x_{1}\right) \geq(1+\Delta) \max \left(f_{2}\left(x_{2}\right), \cdots, f_{n}\left(x_{n}\right)\right)
$$

Similar capture model can be found in [7] (i.e., protocol model). The quantity $\Delta$ models situations where a guard zone is specified to prevent interference. Note also that the capture rule used in the two users' case can be viewed as a special case the above capture rule.

We start with each user facing the same average power constraint (i.e., $e_{1}=e_{2}=\cdots=e_{n}$ ). Since users are identical, it is reasonable to seek a symmetric Nash equilibrium power allocation strategy. Specifically, the set of strategies $\left(g_{1}=g, \cdots, g_{n}=g\right)$ is said to be a symmetric Nash equilibrium strategies if $g_{i}=g$ is the best power allocation strategy for user $i$ when all other users are also employing the power allocation strategy $g$. For a power allocation function $g$ to be a symmetric Nash equilibrium strategy, $f(x)=x g(x)$ must be a strictly increasing function using a similar argument as in the two users case. The following theorem shows the existence and the form of a symmetric Nash equilibrium power allocation strategy.

Theorem 2: Given that each user has the same average power constraint, there exists a symmetric Nash equilibrium power allocation strategy with the following form:

$$
g_{i}\left(x_{i}\right)=c \cdot x_{i}^{n-1} \quad \forall i \in\{1, \cdots, n\}
$$

where $c$ is chosen such that the average power constraint is satisfied.

Proof: The complete proof is given in the Appendix.

With the symmetric Nash equilibrium power allocation strategy given in Eq.(19), the expected throughput for each user is given by:

$$
\begin{aligned}
& P\left(f\left(X_{1}\right) \geq(1+\Delta) \max \left(f\left(X_{2}\right), \cdots, f\left(X_{n}\right)\right)\right) \\
& =P\left(X_{1}^{n} \geq(1+\Delta) \max \left(X_{2}^{n}, \cdots, X_{n}^{n}\right)\right) \\
& =P\left(X_{1} \geq(1+\Delta)^{\frac{1}{n}} \max \left(X_{2}, \cdots, X_{n}\right)\right)
\end{aligned}
$$

To quantify the loss of efficiency due to users' selfish behavior, we consider a system where all users implement the same power allocation policy provided by a system designer such that the overall system throughput is maximized. To find such scheme, we solve the following opti- 
mization problem as in the two users' case:

$$
\begin{aligned}
\max _{v \in S_{1}} P\left(X_{1} v\left(X_{1}\right) \geq\right. & (1+\Delta) \\
& \cdot \max \left(X_{2} v\left(X_{2}\right), \cdots, X_{n} v\left(X_{n}\right)\right)
\end{aligned}
$$

By symmetry, we have the following upper bound for the above probability:

$$
\begin{gathered}
P\left(X_{1} v\left(X_{1}\right) \geq(1+\Delta) \cdot \max \left(X_{2} v\left(X_{2}\right), \cdots,\right.\right. \\
\left.X_{n} v\left(X_{n}\right)\right) \\
<\frac{1}{n}
\end{gathered}
$$

As in the two users' case, we consider a series of functions, $v_{m}(x)=x^{m}$ for $m \geq 1$. As $m \rightarrow \infty$, we have

$$
\begin{aligned}
& P\left(X_{1}^{m+1} \geq(1+\Delta) \cdot \max \left(X_{2}^{m+1}, \cdots, X_{n}^{m+1}\right)\right) \\
& =P\left(X_{1} \geq(1+\Delta)^{\frac{1}{m+1}} \max \left(X_{2}, \cdots, X_{n}\right)\right) \rightarrow \frac{1}{n}
\end{aligned}
$$

Thus, there indeed exists a power allocation scheme that will achieve the maximum possible throughput. In other words, it is possible to have a packet successfully captured in every time slot. Now, when users behave selfishly, the expected throughput for each user is given as follows from Eq.(20):

$$
P\left(X_{1} \geq(1+\Delta)^{\frac{1}{n}} \max \left(X_{2}, \cdots, X_{n}\right)\right)
$$

As $n$ increases, the above equation goes to $1 / n$ which is the maximum attainable throughput. Therefore, as the number of users becomes large, the symmetric Nash equilibrium power allocation scheme is optimal in the sense that the throughput obtained approaches the maximum attainable throughput.

For the special case where $\Delta=0$, the capture rule becomes that the user with the largest received power get captured. With this simple rule, a Nash equilibrium strategy can be derived with general channel state distribution (i.e., $X_{i}$ has probability density function $p_{X_{i}}(\cdot)$ ). From Eq.(37), we have

$$
\begin{aligned}
& p_{Z}\left(f^{-1}\left(x_{1} \cdot b\right)\right) \frac{x_{1}}{f^{\prime}\left(f^{-1}\left(x_{1} \cdot b\right)\right)}=c \\
& f^{\prime}\left(x_{1}\right)=\frac{1}{c} x_{1} p_{Z}\left(x_{1}\right)
\end{aligned}
$$

where

$$
p_{Z}(z)=(n-1) p_{X_{1}}(z)\left[\int_{0}^{z} p_{X_{1}}(x) d x\right]^{n-2} .
$$

Hence, we can write the received power function as the following:

$$
f(x)=\frac{1}{c} \int x p_{Z}(x) d x
$$

From the above equation, one can get the optimal power allocation function by using $g(x)=\frac{f(x)}{x}$.

\section{CONCLUSion}

In this paper, we consider a communication system with multiple users competing for the access of a single satellite, or base station. With a specified capture rule and an average power constraint, users opportunistically adjust their transmission power based on their own channel state to maximize their own individual throughput. Nash equilibrium power allocation strategy is characterized, and the resulting throughput efficiency loss is quantified. As the number of users increases, the Nash equilibrium power allocation strategy approaches the optimal power allocation strategy.

In our analysis of the Nash equilibrium power allocation algorithm, users are assumed to be backlogged. In practice, such assumption may not be appropriate. However, when users do not have data for transmission, one can viewed it as a particular user having a bad channel. Thus, it will be interesting to see whether a Nash equilibrium exists for a channel distribution with significant probability been zero.

\section{APPENDIX}

\section{Proof of Lemmma 2}

Proof: We first show that if $\left(g_{1}^{*}, g_{2}^{*}\right)$ is a Nash equilibrium strategy pair then $D u_{1}\left(x_{1}, g_{1}^{*}\left(x_{1}\right)\right)$ and $D u_{2}\left(x_{2}, g_{2}^{*}\left(x_{2}\right)\right)$ must be constants for all $x_{1} \in[0,1]$ and $x_{2} \in[0,1]$. From user 1's perspective with $g_{2}^{*}$ fixed, consider a small variation of the function $g_{1}^{*}$. Specifically, let $g_{\delta}=g_{1}^{*}+\delta\left(\hat{g}-g_{1}^{*}\right)$ where $\hat{g}$ is an arbitrary function in $S_{1}$. Since both $\hat{g}$ and $g_{1}^{*}$ are in $S_{1}$, they are both bounded (i.e., $\left|\hat{g}\left(x_{1}\right)\right| \leq B$ and $\left|g_{1}^{*}\left(x_{1}\right)\right| \leq B$ for all $\left.x_{1} \in[0,1]\right)$. Therefore, we have $\left|g_{\delta}\left(x_{1}\right)-g_{1}^{*}\left(x_{1}\right)\right| \leq 2 B \delta$ for all $x_{1} \in[0,1]$. Using the Lagrange's form of Taylor's theorem, we get for any $x_{1} \in[0,1]$, there exists a real number $c_{\left[x_{1}\right]} \in\left[g_{1}^{*}\left(x_{1}\right), g_{\delta}\left(x_{1}\right)\right]$ such that

$$
\begin{aligned}
& u_{1}\left(x_{1}, g_{\delta}\left(x_{1}\right)\right)=u_{1}\left(x_{1}, g_{1}^{*}\left(x_{1}\right)\right) \\
& \quad+\left.\delta\left(\hat{g}\left(x_{1}\right)-g_{1}^{*}\left(x_{1}\right)\right) \frac{\partial u_{1}\left(x_{1}, b\right)}{\partial b}\right|_{b=g_{1}^{*}\left(x_{1}\right)} \\
& \quad+\left.\frac{1}{2} \delta^{2}\left(\hat{g}\left(x_{1}\right)-g_{1}^{*}\left(x_{1}\right)\right)^{2} \frac{\partial^{2} u_{1}\left(x_{1}, b\right)}{\partial b^{2}}\right|_{b=c_{\left[x_{1}\right]}}
\end{aligned}
$$

The last term is bounded by $K \cdot \delta^{2}$ for some $K$ since both $\hat{g}$ and $g_{1}^{*}$ are bounded, and $u_{1}\left(x_{1}, b\right)$ has finite second derivative. Therefore, for small enough $\delta$, it is negligible comparing with the other terms.

Now we show that if $D u_{1}\left(x_{1}, g_{1}^{*}\left(x_{1}\right)\right)$ is not a constant for all $x_{1} \in[0,1]$, we can find a strategy $g_{\delta}$ which gives user 1 a higher throughput than $g_{1}^{*}$. To do that, we can 
write the following equations:

$$
\begin{aligned}
& \int_{0}^{1} u_{1}\left(x_{1}, f_{\delta}\left(x_{1}\right)\right) d x_{1}-\int_{0}^{1} u_{1}\left(x_{1}, f_{1}^{*}\left(x_{1}\right)\right) d x_{1} \\
& =\left.\delta \int_{0}^{1}\left(\hat{g}\left(x_{1}\right)-g_{1}^{*}\left(x_{1}\right)\right) \frac{\partial u_{1}\left(x_{1}, b\right)}{\partial b}\right|_{b=g_{1}^{*}\left(x_{1}\right)} d x_{1} \\
& \quad+o(\delta)
\end{aligned}
$$

Now, since $D u_{1}\left(x_{1}, g_{1}^{*}\left(x_{1}\right)\right)$ is not a constant for all $x_{1} \in$ $[0,1]$, we can find a $\hat{g}$ such that the above equation is positive which implies that there is an incentive for user 1 to use $g_{\delta}$. Hence, $\left(g_{1}^{*}, g_{2}^{*}\right)$ is not a Nash equilibrium strategy pair. Similarly, we can show that $D u_{2}\left(x_{2}, g_{2}^{*}\left(x_{2}\right)\right)$ is a constant for all $x_{2} \in[0,1]$ if $\left(g_{1}^{*}, g_{2}^{*}\right)$ is a Nash equilibrium strategy pair.

For the converse, consider again Eq.(24). Since $D u_{1}\left(x_{1}, g_{1}^{*}\left(x_{1}\right)\right)=\left.\frac{\partial u_{1}\left(x_{1}, b\right)}{\partial b}\right|_{b=g_{1}^{*}\left(x_{1}\right)}$ equals to a constant $c_{1}$ for all $x_{1} \in[0,1]$. We have

$$
\begin{aligned}
& \left.\delta \int_{0}^{1}\left(\hat{g}\left(x_{1}\right)-g_{1}^{*}\left(x_{1}\right)\right) \frac{\partial u_{1}\left(x_{1}, b\right)}{\partial b}\right|_{b=g_{1}^{*}\left(x_{1}\right)} d x_{1} \\
& =\delta c_{1} \int_{0}^{1}\left(\hat{g}\left(x_{1}\right)-g_{1}^{*}\left(x_{1}\right)\right) d x_{1}=0
\end{aligned}
$$

for all $\hat{g} \in S_{1}$ (i.e., $\int_{0}^{1} \hat{g}\left(x_{1}\right) d x_{1}=e_{1}$ ). Thus, there is no incentive for user 1 to use strategy $\hat{g}$. Therefore, $\left(g_{1}^{*}, g_{2}^{*}\right)$ is a Nash equilibrium strategy pair.

\section{Proof of Corollary}

We have established that $g_{1}^{*}(1)=g_{2}^{*}(1)$ is a necessary condition for $\left(g_{1}^{*}, g_{2}^{*}\right)$ to be an equilibrium strategy pair from lemma 3. Combining with $g_{1}^{*}(0)=g_{2}^{*}(0)=0$, we will consider only the function pair $g_{1} \in S_{1}$ and $g_{2} \in$ $S_{2}$ that satisfy the above two boundary conditions (i.e., $g_{1}(1)=g_{2}(1)$ and $\left.g_{1}(0)=g_{2}(0)=0\right)$.

Consider any Nash equilibrium strategy pair $\left(g_{1}, g_{2}\right)$ under the capture rule described in the two users' case. From previous discussion, we know that the inverse functions, $f_{2}^{-1}$ and $f_{1}^{-1}$ where $f_{1}=x g_{1}(x)$ and $f_{2}=x g_{2}(x)$, are well defined. With user 2's strategy $g_{2}$ fixed, we have

$$
u_{1}\left(x_{1}, b\right)=P\left(f_{2}\left(X_{2}\right) \leq x_{1} \cdot b\right)=f_{2}^{-1}\left(x_{1} \cdot b\right)
$$

Similarly, with user1's strategy $f_{1}$ fixed, we get

$$
u_{2}\left(x_{2}, b\right)=P\left(f_{1}\left(X_{1}\right) \leq x_{2} \cdot b\right)=f_{1}^{-1}\left(x_{2} \cdot b\right)
$$

From Lemma 2, we know that $D u_{1}\left(x_{1}, g_{1}\left(x_{1}\right)\right)$ and $D u_{2}\left(x_{2}, g_{2}\left(x_{2}\right)\right)$ are two constants for all $x_{1} \in[0,1]$ and $x_{2} \in[0,1]$ since $\left(g_{1}, g_{2}\right)$ is a Nash equilibrium strategy pair.
Now, consider the set of channel state pair $\left(x_{1}, x_{2}\right)$ such that $f_{1}\left(x_{1}\right)=f_{2}\left(x_{2}\right)$ (i.e., two users' received power are equal). It forms a separation line in space span by $X_{1}$ and $X_{2}$. Mathematically, this line can be defined as $h:[0,1] \rightarrow[0,1]$ such that $x_{2}=h\left(x_{1}\right)=f_{2}^{-1}\left(f_{1}\left(x_{1}\right)\right)$. By the capture rule, a slot with channel state $\left(x_{1}, x_{2}^{\prime}\right)$ will be successfully used by user 2 if $\left(x_{1}, x_{2}^{\prime}\right)$ is above the line $x_{2}=h\left(x_{1}\right)$ and by user 1 if $\left(x_{1}, x_{2}^{\prime}\right)$ is below the separation line. Fig. 2 shows an example of $h\left(x_{1}\right)$. The following lemma shows the uniqueness of $h\left(x_{1}\right)$. We then derive the uniqueness of the strategy pair $\left(g_{1}, g_{2}\right)$ from the lemma.

Lemma 4: If $D u_{1}\left(x_{1}, g_{1}\left(x_{1}\right)\right)$ and $D u_{2}\left(x_{2}, g_{2}\left(x_{2}\right)\right)$ are two constants, $c_{1}$ and $c_{2}$ respectively, for all $x_{1} \in[0,1]$ and $x_{2} \in[0,1]$, then $h\left(x_{1}\right)=x_{1}^{c_{1} / c_{2}}$.

Proof: $\quad$ Since $D u_{1}\left(x_{1}, g_{1}\left(x_{1}\right)\right)=c_{1}$, from $u_{1}\left(x_{1}, b\right)=f_{2}^{-1}\left(x_{1}, b\right)$, we have

$$
\begin{aligned}
D u_{1}\left(x_{1}, g_{1}\left(x_{1}\right)\right) & =\left.\frac{\partial u_{1}\left(x_{1}, b\right)}{\partial b}\right|_{b=g_{1}\left(x_{1}\right)} \\
& =\frac{x_{1}}{f_{2}^{\prime}\left(f_{2}^{-1}\left(x_{1} \cdot g_{1}\left(x_{1}\right)\right)\right)} \\
& =\frac{x_{1}}{f_{2}^{\prime}\left(f_{2}^{-1}\left(f_{1}\left(x_{1}\right)\right)\right)}=c_{1} \\
f_{2}^{\prime}\left(h\left(x_{1}\right)\right) & =\frac{x_{1}}{c_{1}}
\end{aligned}
$$

Similarly, for user 2, we get

$$
\begin{aligned}
D u_{2}\left(x_{2}, f_{2}\left(x_{2}\right)\right) & =\left.\frac{\partial u_{2}\left(x_{2}, b\right)}{\partial b}\right|_{b=g_{2}\left(x_{2}\right)} \\
& =\frac{x_{2}}{f_{1}^{\prime}\left(f_{1}^{-1}\left(f_{2}\left(x_{2}\right)\right)\right)}=c_{2} \\
f_{1}^{\prime}\left(h^{-1}\left(x_{2}\right)\right) & =\frac{x_{2}}{c_{2}}
\end{aligned}
$$

We also know that $f_{1}\left(x_{1}\right)=f_{2}\left(h\left(x_{1}\right)\right)$ and $f_{1}^{\prime}\left(x_{1}\right)=$ $f_{2}^{\prime}\left(h\left(x_{1}\right)\right) \cdot h^{\prime}\left(x_{1}\right)$. Thus, we have

$$
\begin{aligned}
f_{1}^{\prime}\left(h^{-1}\left(x_{2}\right)\right) & =f_{2}^{\prime}\left(h\left(h^{-1}\left(x_{2}\right)\right)\right) \cdot h^{\prime}\left(h^{-1}\left(x_{2}\right)\right) \\
& =f_{2}^{\prime}\left(x_{2}\right) \cdot h^{\prime}\left(x_{1}\right) \\
& =f_{2}^{\prime}\left(h\left(x_{1}\right)\right) \cdot h^{\prime}\left(x_{1}\right)
\end{aligned}
$$

By combining the equations $f_{1}^{\prime}\left(h^{-1}\left(x_{2}\right)\right)=\frac{x_{2}}{c_{2}}$ and $f_{1}^{\prime}\left(h^{-1}\left(x_{2}\right)\right)=f_{2}^{\prime}\left(h\left(x_{1}\right)\right) \cdot h^{\prime}\left(x_{1}\right)$, we get

$$
\frac{x_{2}}{c_{2}}=f_{2}^{\prime}\left(h\left(x_{1}\right)\right) \cdot h^{\prime}\left(x_{1}\right) .
$$

Next we substitute Eq.(26) and $x_{2}=h\left(x_{1}\right)$ in the above 
equation to obtain,

$$
\begin{aligned}
x_{1} \cdot \frac{d h\left(x_{1}\right)}{d x_{1}} & =\frac{c_{1}}{c_{2}} h\left(x_{1}\right) \\
\frac{d h\left(x_{1}\right)}{h\left(x_{1}\right)} & =\frac{c_{1}}{c_{2}} \frac{d x_{1}}{x_{1}} \\
\ln \left|h\left(x_{1}\right)\right| & =\frac{c_{1}}{c_{2}} \ln \left|x_{1}\right|+c_{3} \\
h\left(x_{1}\right) & =e^{c_{3}} \cdot x_{1}^{\frac{c_{1}}{c_{2}}}
\end{aligned}
$$

Combined with fact that $h(1)=1$, we get $h\left(x_{1}\right)=x_{1}^{\frac{c_{1}}{c_{2}}}$.

Now, we are in a position to derive the exact form of the Nash equilibrium strategy pair. From the equations $f_{1}^{\prime}\left(h^{-1}\left(x_{2}\right)\right)=\frac{x_{2}}{c_{2}}$ and $x_{2}=h\left(x_{1}\right)$, we get $f_{1}^{\prime}\left(x_{1}\right)=$ $\frac{h\left(x_{1}\right)}{c_{2}}=x_{1}^{\frac{c_{1}}{c_{2}}} / c_{2}$. Combined with the condition that $f_{1}(0)=0$, we have $f_{1}(x)=\frac{1}{c_{1}+c_{2}} x^{\frac{c_{1}}{c_{2}}+1}$. Following the similar method, we get $f_{2}(x)=\frac{1}{c_{1}+c_{2}} x^{\frac{c_{2}}{c_{1}}+1}$. Let $\gamma=\frac{c_{1}}{c_{2}}$ and $c=\frac{1}{c_{1}+c_{2}}$, the received power of a Nash equilibrium strategy pair must have the following form:

$$
\begin{aligned}
& f_{1}^{*}\left(x_{1}\right)=c \cdot x_{1}^{\gamma+1} \\
& f_{2}^{*}\left(x_{2}\right)=c \cdot x_{1}^{\frac{1}{\gamma}+1}
\end{aligned}
$$

Consequently, we have the Nash equilibrium power allocation strategy to be the form:

$$
\begin{aligned}
& g_{1}^{*}\left(x_{1}\right)=c \cdot x_{1}^{\gamma} \\
& g_{2}^{*}\left(x_{2}\right)=c \cdot x_{1}^{\frac{1}{\gamma}}
\end{aligned}
$$

The constant $\gamma$ and $c$ are chosen such that equations (11) and (12) are satisfied. The uniqueness of the above Nash equilibrium strategy comes from the fact that there is a unique pair of $c$ and $\gamma$ that satisfy equations (11) and (12).

\section{Proof of Theorem 2}

Proof: With all users $i \neq 1$ using a fixed power allocation strategy $g$, we now explore the optimal power allocation strategy for user 1 which is denoted by $g_{1}^{*}$. Let $u_{g}^{(1)}:\left(x_{1}, b\right) \rightarrow \mathcal{R}$ denote user 1's expected throughput during a slot conditioning on the following events:

- User 1's channel state is $X_{1}=x_{1}$.

- User 1's allocated power is $b$.

As before, we will drop the term $g$ in the expression $u_{g}^{(1)}\left(x_{1}, b\right)$, and simply write it as $u_{1}\left(x_{1}, b\right)$. Specifically, we can the write the equation:

$$
\begin{aligned}
& u_{1}\left(x_{1}, b\right) \\
= & P\left((1+\Delta) \max \left(f_{2}\left(X_{2}\right), \cdots, f_{n}\left(X_{n}\right)\right) \leq x_{1} \cdot b\right) \\
= & P\left((1+\Delta) Y \leq x_{1} \cdot b\right)
\end{aligned}
$$

where $Y=\max \left(f_{2}\left(X_{2}\right), \cdots, f_{n}\left(X_{n}\right)\right)$. Since all users $i \neq 1$ use the same strategy $g$, we have $Y=$ $\max \left(f\left(X_{2}\right), \cdots, f\left(X_{n}\right)\right)$ where $f\left(X_{i}\right)=X_{i} \cdot g\left(X_{i}\right)$ for all $i \neq 1$. Moreover, since $f$ is strictly increasing, we can write:

$Y=\max \left(f\left(X_{2}\right), \cdots, f\left(X_{n}\right)\right)=f\left(\max \left(X_{2}, \cdots, X_{n}\right)\right)$

Denoting $Z=\max \left(X_{2}, \cdots, X_{n}\right)$, we have the following:

$$
\begin{aligned}
u_{1}\left(x_{1}, b\right) & =P\left((1+\Delta) Y \leq x_{1} \cdot b\right) \\
& =P\left(Z \leq f^{-1}\left(\frac{1}{1+\Delta} x_{1} \cdot b\right)\right) \\
& =\int_{0}^{f^{-1}\left(\frac{1}{1+\Delta} x_{1} \cdot b\right)} p_{Z}(z) d z
\end{aligned}
$$

where $p_{Z}(\cdot)$ denote the probability density function of the random variable $Z$. The optimization problem that user 1 faces can be written as the following:

$$
\begin{aligned}
\max G_{1}(e) & =\int_{0}^{1} u_{1}\left(x_{1}, g_{1}\left(x_{1}\right)\right) \cdot p_{X_{1}}\left(x_{1}\right) d x_{1} \\
& =\int_{0}^{1} u_{1}\left(x_{1}, g_{1}\left(x_{1}\right)\right) d x_{1} \\
\text { subj. } & \int_{0}^{1} g_{1}\left(x_{1}\right) d x_{1} \leq e
\end{aligned}
$$

Writing the Lagrangian function, we have

$$
\begin{aligned}
& \int_{0}^{1} u_{1}\left(x_{1}, g_{1}\left(x_{1}\right)\right) d x_{1}-\lambda\left(\int_{0}^{1} g_{1}\left(x_{1}\right) d x_{1}-e\right) \\
= & \int_{0}^{1}\left[u_{1}\left(x_{1}, g_{1}\left(x_{1}\right)\right)-\lambda g_{1}\left(x_{1}\right)\right] d x_{1}+\lambda e
\end{aligned}
$$

Therefore, for each fixed $x_{1}$, we want to choose a $g_{1}\left(x_{1}\right)$ to maximize the term $u_{1}\left(x_{1}, g_{1}\left(x_{1}\right)\right)-\lambda g_{1}\left(x_{1}\right)$. For convenience, let $b=g_{1}\left(x_{1}\right)$. Then, we have

$$
\begin{aligned}
& \max _{b} L(b)=u_{1}\left(x_{1}, b\right)-\lambda b \\
= & \max _{b} \int_{0}^{f^{-1}\left(\frac{1}{1+\Delta} x_{1} \cdot b\right)} p_{Z}(z) d z-\lambda b
\end{aligned}
$$

Maximizing $L(b)$ with respect to $b$ yields the first order condition:

$$
\begin{aligned}
\frac{\partial L(b)}{\partial b}= & p_{Z}\left(f^{-1}\left(\frac{1}{1+\Delta} x_{1} \cdot b\right)\right) \frac{\frac{x_{1}}{1+\Delta}}{f^{\prime}\left(f^{-1}\left(\frac{1}{1+\Delta} x_{1} \cdot b\right)\right)} \\
& -\lambda=0
\end{aligned}
$$


Since $Z=\max \left(X_{2}, \cdots, X_{n}\right)$ and $X_{i}$ 's are i.i.d, we have

$$
p_{Z}(z)=(n-1) z^{n-2} .
$$

Now, consider $b=g_{1}\left(x_{1}\right)=c x_{1}^{m}$. Since we are seeking a symmetric Nash equilibrium power allocation strategy, user $i \neq 1$ will adopt the same strategy as user 1 . Thus, we have $f(x)=x \cdot g(x)=x \cdot c x^{m}=c x^{m+1}$. The second term in Eq.(37) can be written as the following:

$$
\begin{aligned}
& f^{\prime}\left(f^{-1}\left(\frac{1}{1+\Delta} x_{1} \cdot b\right)\right) \\
& =f^{\prime}\left(f^{-1}\left(\frac{c}{1+\Delta} x_{1} \cdot x_{1}^{m}\right)\right) \\
& =f^{\prime}\left(\left(\frac{1}{1+\Delta} x_{1} \cdot x_{1}^{m}\right)^{\frac{1}{m+1}}\right) \\
& =c(m+1)\left(\frac{1}{1+\Delta}\right)^{\frac{m}{m+1}} x_{1}^{m}
\end{aligned}
$$

Similarly,

$$
\begin{aligned}
& p_{Z}\left(f^{-1}\left(\frac{1}{1+\Delta} x_{1} \cdot b\right)\right) \\
& =p_{Z}\left(\left(\frac{1}{1+\Delta}\right)^{\frac{1}{m+1}} x_{1}\right) \\
& =(n-1)\left(\frac{1}{1+\Delta}\right)^{\frac{n-2}{m+1}} x_{1}^{n-2}
\end{aligned}
$$

Eq.(37) can be re-written in the following form:

$$
(n-1)\left(\frac{1}{1+\Delta}\right)^{\frac{n-2}{m+1}} x_{1}^{n-2} \frac{\frac{x_{1}}{1+\Delta}}{c(m+1)\left(\frac{1}{1+\Delta}\right)^{\frac{m}{m+1}} x_{1}^{m}}-\lambda=0
$$

Since the above equality has to hold for all $x_{1} \in[0,1]$, the following must be true

$$
x_{1}^{n-2} \cdot x_{1} \cdot x_{1}^{-m}=1
$$

Thus, we have $m=n-1$ and $g_{i}(x)=c x^{n-1}$ for all $i=1, \cdots, n$.

\section{REFERENCES}

[1] A. Fu, E. Modiano, and J. Tsitsiklis, "Optimal energy allocation for delay-constrained data transmission over a time-varying channel," IEEE INFOCOM 2003, San Francisco, CA, April 2003.

[2] A. MacKenzie and S. Wicker, "Stability of multipacket slotted Aloha with selfish users and perfect information," IEEE INFOCOM 2003, San Francisco, CA, Mar. 2003.

[3] X. Qin and R. Berry, "Exploiting Multiuser Diversity for Medium Access Control in Wireless Networks," IEEE INFOCOM 2003, San Francisco, CA, Mar. 2003.

[4] S. Ghez, S. Verdu, and S. Schwartz, "Stability properties of slotted Aloha with multipacket reception capability," IEEE Tran. on Automatic Control, vol. 33, no. 7, pp. 640-649, July 1988.

[5] N. Abramson, "The throughput of packet broadcasting channels," IEEE Tran. on Communications, vol. COM-25, pp. 117-128, 1977.
[6] P. Viswanath, D. Tse, and R. Laroia, "Opportunistic beamforming using dumb antennas," IEEE Tran. on Information Theory, vol. 48, no. 6, pp. 1277-1294, June 2002.

[7] P. Gupta and P. R. Kumar, "The capacity of wireless networks," IEEE Tran. on Information Theory, vol. 46, no. 2, pp. 388-404, Mar. 2000.

[8] W. Luo and A. Ephremides, "Power levels and packet lengths in random multiple access," IEEE Tran. on Information Theory, vol. 48, no. 1, pp. 46-58, Jan. 2002.

[9] E. Altman, V. Borkar and A. A. Kherani, "Optimal random access in networks with two-way traffic," The 15th IEEE International Symposium on Personal, Indoor and Mobile Radio Communications (PIMRC 2004), Barcelona, Spain, 5-8, Sept., 2004.

[10] D. Bertsekas and R. Gallager, Data Networks, Prentice Hall, 1991.

[11] R. Johari and J. Tsitsiklis, " Network resource allocation and a congestion game," submitted May 2003.

[12] P. Klemperer, "Auction theory: A guide to the literature," Journal of Economics Surveys, vol. 13(3), pp. 227-286, July 1999.

[13] Y-K. Che and I. Gale, "Standard auctions with financially constrained bidders," Review of Economic Studies, vol 65, pp. 1-21, January 1998.

[14] T. R. Palfrey, "Multiple-object, discriminatory auctions with bidding constraints: A game-theoretic analysis," Management Scienece, vol 26, pp. 935-946, September 1980.

[15] D. Famolari, N. Mandayam, and D. Goodman, "A new framework for power control in wireless data networks: games, utility, and pricing," Allerton Conference on Communication, Control, and Computing, Monticello, IL, September 1998.

[16] T. Basar and R. Srikant, "Revenue-maximizing pricing and capacity expansion in a many-users regime," IEEE INFOCOM 2002, vol. 1, pp. 23-27, June 2002. 